\title{
Ship Target Movement Parameter Estimation Based on Three Linear Array High-resolution Remote Sensing Image
}

\author{
Yongzhou $\mathrm{Lu}^{1, \mathrm{a}}$, Hongbing $\mathrm{Ma}^{2, \mathrm{~b}}$ \\ 1,2Department of Electronic Engineering of Tsinghua University, Beijing, 100062, \\ China ${ }^{2} l y z$ navy@163.com, 'bhbma@mail.tsinghua.edu.cn
}

Keywords: high-resolution satellite images; double threshold detection; three linear CCD, ship velocity.

\begin{abstract}
Aiming at rapid ship target detection and movement parameter estimation in highresolution satellite image, this paper firstly analyzes marine and continental background image features. Furthermore, based on pixel gradient extraction and edge detection techniques, this paper extracts the ship target with the gradient map-based double threshold segmentation detection method. On the basis of utilizing three linear CCD shooting function of ALOS satellite, it finally proposes the method to rapidly estimate the movement parameter of the target ship by taking advantages of the long shooting interval.
\end{abstract}

\section{Introduction}

Along with rapid development of high-resolution satellite, detection and parameter estimation against the moving target by high-resolution visible images gradually becomes a focus of study. How to rapidly acquire movement parameter of suspicious target has great military significance on our security defense.

Among domestic and overseas studies on detection and movement parameter estimation of the moving ship target, a majority focuses on use of the methods such as SAR satellite images, GPSlocators measurement or sound intensity vector measurement[3,4,5,6], while studies on moving target parameter estimation by using visible images are relatively fewer. Literature [1] proposes that recording time difference of QUICKBIRD panchromatic / multispectral image can be used to fuse the pixel of two images. The images fused will produce double images against the moving target and parameter estimation of the target can be carried out by calculating the number of pixels of double images. In literature [2], the ship velocity is calculated by using kelvin-bridge to extract transverse wavelength of wake stream of the ship during movement, namely Kelvin wake. Among the above literatures, the method proposed in literature [1] requires a series of image fusion calculation, which is too cumbersome. Furthermore, due to short shooting interval of QUICKBIRD, only high-speed targets including automotive and aircrafts can be processed by parameter estimation. As for method in literature [2], image quality is stressed and the error is relatively large.

This paper proposes the method that is to estimate the parameters of the target ship movement by using three stereograms taken by ALOS satellite. After extracting ocean background from three calibrated stereograms and detecting the interested moving target, it finally achieves movement parameter estimation of target ship by using the feature of long shooting interval.

\section{Target ship detection}

The In the typical marine and continental background images, there are obvious land and sea features. The sea areas show gentle gray change and uniform gray distribution, while the land areas show dramatic gray change and large gray distribution span. Thus, after obtaining of image gradient value with Sobel operator, the land and the sea can be separated by using double threshold detection method and the ship target can be detected with canny operator after extraction of sea areas. Finally, satellite three-stereogram shooting principle is utilized to estimate the velocity parameter of the moving ship target. Specific flow chart goes as follows: 


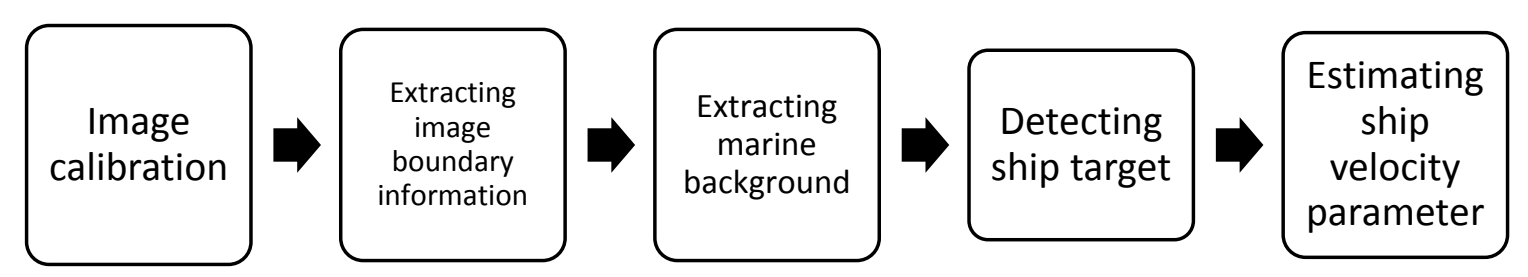

Fig 1 Specific flow chart

\subsection{Double threshold segmentation detection method}

In satellite images with marine and continental background, the continental background has distinct complex boundary image, while the marine background has weak boundary image. Thus, the double threshold segmentation detection method is selected in this paper. Firstly, the Sobel operator is used to obtain image gradient, followed by use of two thresholds with different levels for image processing. Then, the connected domain of nonzero pixels corresponding to low threshold image is found by using the high threshold image and land areas are recognized with corrosion and dilation method to achieve segmentation of land and sea.

Sobel operator is used to obtain gradient magnitude, which is considered as boundary image. 3 $\times 3$ template is selected to calculate the gradient value in each pixel, with the formula going as follows:

\begin{tabular}{|c|c|c|}
\hline$I_{1}$ & $I_{2}$ & $I_{3}$ \\
\hline$I_{4}$ & $(\mathrm{x}, \mathrm{y})$ & $I_{5}$ \\
\hline$I_{6}$ & $I_{7}$ & $I_{8}$ \\
\hline
\end{tabular}

Fig 2 Gradient of central point with $3 \times 3$ template

$$
\begin{aligned}
& S_{x}=\left(I_{1}+2 I_{2}+I_{3}\right)-\left(I_{6}+2 I_{7}+I_{8}\right) \\
& S_{y}=\left(I_{1}+2 I_{4}+I_{6}\right)-\left(I_{3}+2 I_{5}+I_{8}\right) \\
& M_{(x, y)}=\sqrt{\left(S_{x}{ }^{2}+S_{y}{ }^{2}\right)}
\end{aligned}
$$

Where, $I_{\mathrm{i}}$, (i=1 $\quad(\ldots . .8)$ is the pixel value in the connected domain around the central point; $S_{x}$ and $S_{y}$ are respectively horizontal and vertical gradients and $M_{(x, y)}$ is the gradient value of the central pint $(x, y)$.

After acquiring the boundary image, the image is processed by double threshold segmentation and two threshold with different values, $T_{1}$ and $T_{2}$, are selected, of which $T_{1}>T_{2}$. The specific steps are as follows:

1) A threshold $T_{1}=0.4$ is set for boundary image. Pixels below $T_{1}$ are filtered out to obtain the high- threshold boundary diagram, where the distinct boundary image of land areas is retained while weaker boundary image of sea areas is filtered out.

2) A threshold $T_{2}=0.035$ is set for boundary image. Due to low threshold, most of boundary images are retained, as shown in Fig. 4;

3) The connected domain corresponding to non-zero pixel in the high threshold map is found out in low threshold map to identify the land areas, as shown in Fig.5; 
4) Land areas are identified after the image is processed by corrosion and dilation. Although the ship target has a certain gradient value, the land areas can be obtained with the ship target reserved because the land area is much larger than the area of ship target to finally acquire marine and continental segmentation image, as shown in Fig. 6

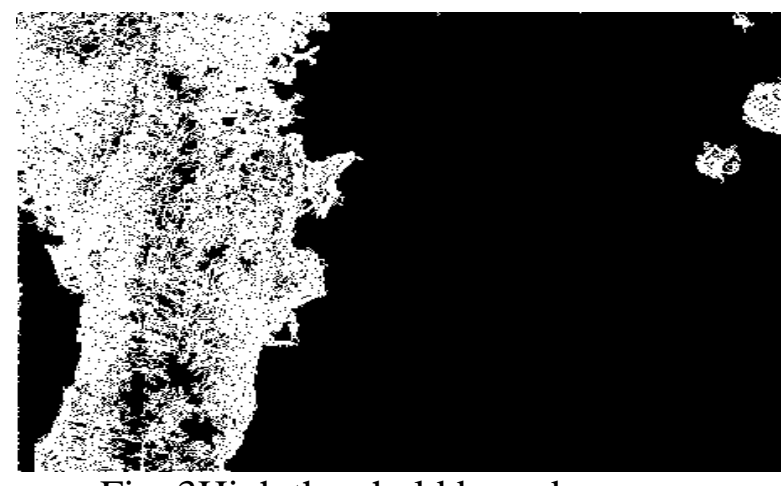

Fig. 3High threshold boundary map

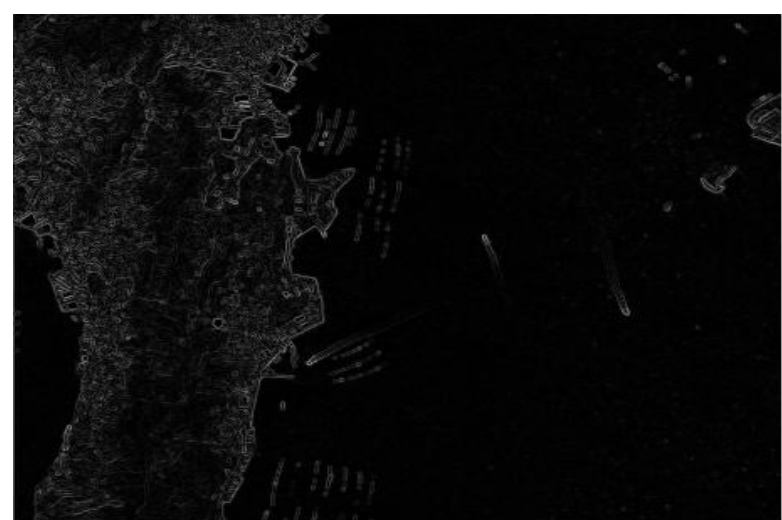

Fig. 5 Land areas map

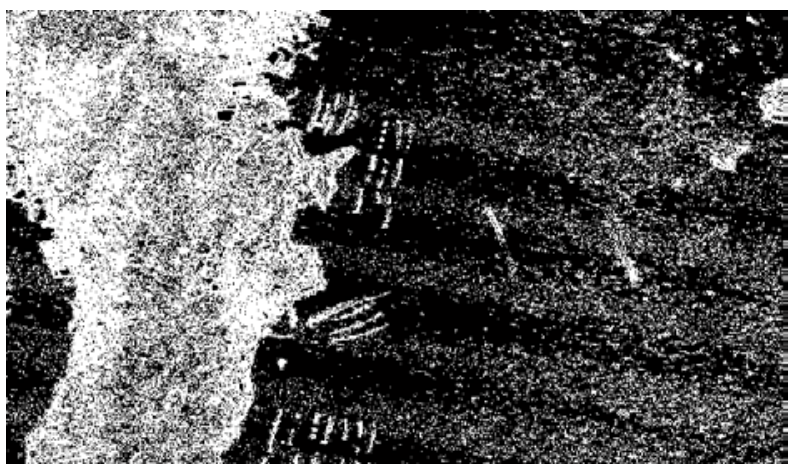

Fig. 4 Low threshold boundary map

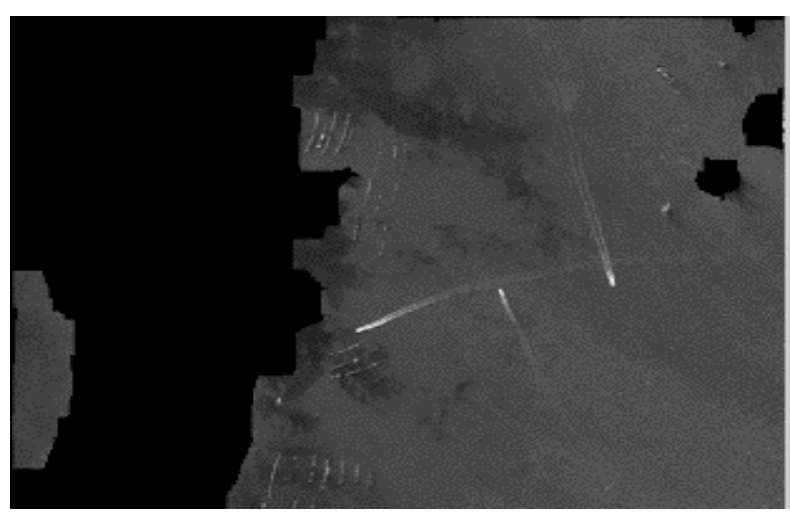

Fig. 6 Result map

\subsection{Ship target extraction}

In the case where the marine background is separated from the continental background, the ship target has been more obvious. However, the wave texture and random noise in the marine background case will affect the detection result of the target, among which the wave texture and the wake have the maximum impact on target detection. Firstly, simple grey filter can be used. The grey threshold is proven to be optimal at 0.7 , when there is a small amount of large noise in the image. Then, Gauss smooth filter is used to better filter out the noisy points. Finally, canny operator boundary detection technique is used to rapidly extract the ship target position, as shown in Fig. 7.

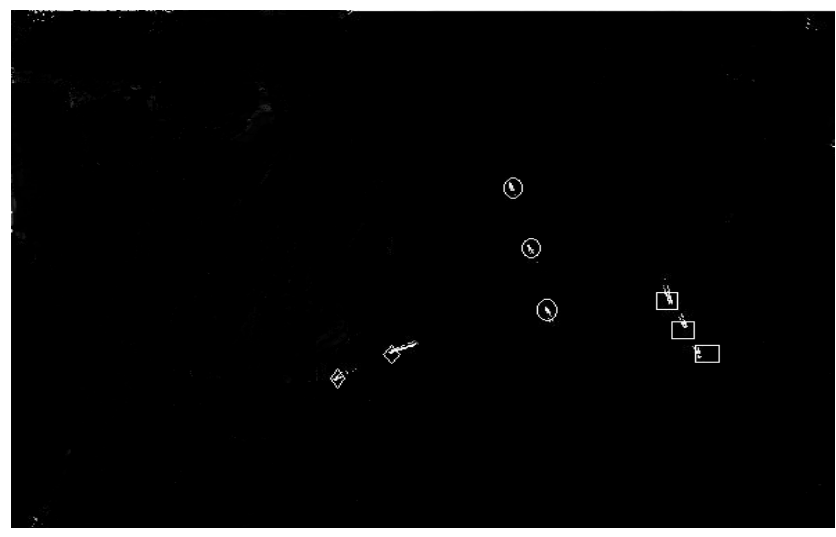

Fig. 7 Ship target detection map 


\section{Ship velocity estimation}

\subsection{ALOS parameters}

ALOS is an earth observing satellite in Japan, which is equipped with panchromatic remote sensing stereotelemeter(PRISM) and is mainly used for digital elevation mapping. PRISM has three independent cameras to observe substellar point, forward and rear points, acquiring threedimensional images along the track. Spatial resolution of substellar point is $2.5 \mathrm{~m}$, and shooting intervals of such three cameras is 10 minutes. When the same area is screened, three displacements will be produced to the moving target. After obtaining the number of pixels of such three displacement with relevant algorithm, velocity of the moving target can be estimated.

\subsection{Satellite image processing}

1) Three stereograms of the same area is extracted in this paper. Due to different shooting angles, grey values will be different. Thus, such three images have to be calibrated. Just as shown in the figure, significant displacement happens.

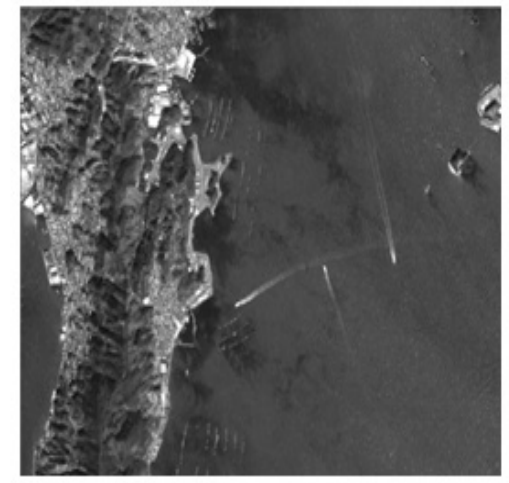

Fig.a'ALOS'forward'view

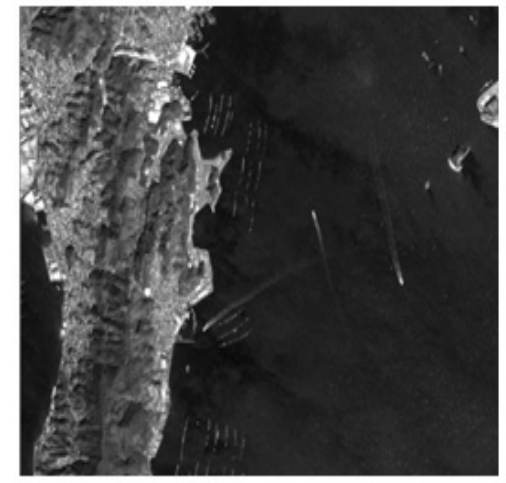

Fig b.ALOS substellar point view

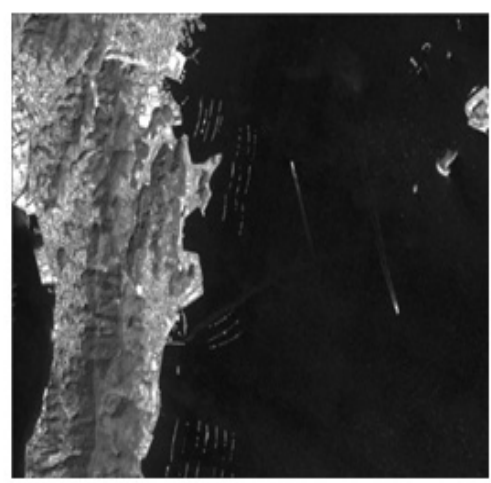

Fig.'c'ALOS'rear'view

2) After calibration, the fore-going detection methods and the following formula are used to process such three views:

$\mathrm{P}=\left\|M_{B}-M_{N}\right\|+M_{F}$

Where $M_{B}, M_{N}, M_{F}$ are pixel matrixes respectively for substellar point, forward and rear views and $\mathrm{P}$ is the processing result.

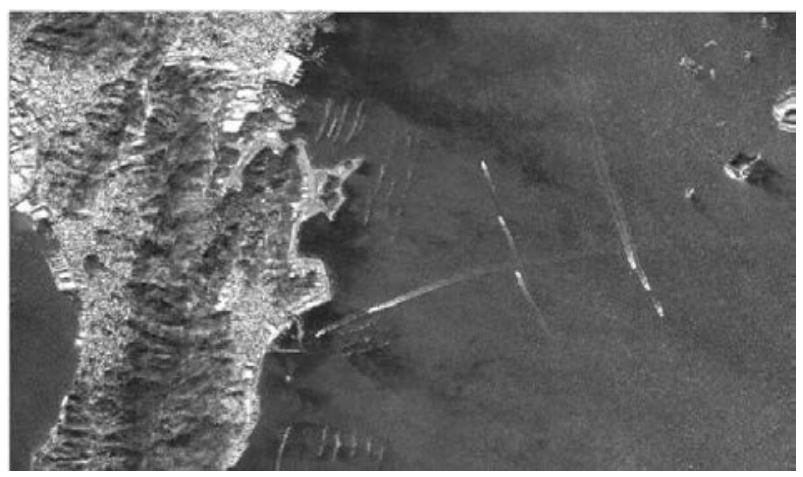

Fig. 8 Three stereograms overlaying map 


\subsection{Ship target velocity parameter estimation}

It can be seen from Fig. 8 that the ship target is well preserved and the distance among each target is clearly visible, which is convenient to estimate the velocity with satellite parameters. According to relevant materials, as the angle of the satellite is \pm 23.8 degree and the shooting height is $691.65 \mathrm{~km}$, the shooting interval difference is 10 minutes. After that, the number of pixels at each displacement of three targets can be calculated with relevant software and the distance is obtained with image resolution to finally estimate the velocity parameter of each target, with results shown as follows:

\begin{tabular}{|l|c|}
\hline \multicolumn{1}{|c|}{ Ship Target } & Speed parameter \\
\hline The left side of the target & $17.62 \mathrm{~km} / \mathrm{h}$ \\
\hline Intermediate target & $26.76 \mathrm{~km} / \mathrm{h}$ \\
\hline The right side of the target & $18.12 \mathrm{~km} / \mathrm{h}$ \\
\hline
\end{tabular}

\section{Conclusion:}

Target parameter estimation of high-resolution remote sensing image has always been a focus of image study. Traditional detection methods tend to be complicated with low practicality. Based on the previous literatures, this paper firstly segments marine and continental backgrounds in the satellite image taken by three line array with double threshold segmentation method and then detects the target with canny operator. Finally, it extracts relevant data after overlapping and processing three views to rapidly and effectively acquire the ship target velocity parameter by combing with satellite parameters. The method proposed in this paper is proven to have high practical value and can provide reference for further high-resolution satellite imagery target parameters estimation.

\section{References}

[1] Zhang Boyan Detection and Speed Estimation of Moving Vehicle from Remote Sensing Images. [D] Xidian university Xian, 2013.

[2] ZENG Lei, WANG Xiaorui, GUO Bingtao, YU Huaxin. A Ship Target Detection and Speed Estimation Method with Space flight Image, [J]. Opto-Electronic Engineering, 2013, 40(12) , 27-52

[3] CRISP D J. The state-of-the-art in ship detection in synthetic aperture radar imagery [R]. DSTO Information Sciences Laboratory, 2004: 26-72.

[4] HENNINGD I, ROMEISER R, ALPERS W, et al. Radarimaging of Kelvin arms and ship wakes [J]. International Journal of Remote Sensing(S0143-1161)，1999，20(13): 2519-2543.

[5] Pierce S D, Barth J A, Smith R L. Improving acoustic Doppler current profiler accuracy with wide-area differential GPS and adaptive smoothing of ship velocity [J]. Journal of Atmospheric and Oceanic Technology(S0739-0572), 1999, 16(5): 591-596.

[6] Denbigh P N. Ship velocity determination by Doppler and correlation techniques [J]. IEE Proceedings, Part F: Communications, Radar and Signal Processing(S0143-7070), 1984, 131(3): 315-326. 